\title{
A amamentação em tempos da COVID-19: uma revisão narrativa
}

RESUMO | Objetivo: Analisar a amamentação em tempos de pandemia da COVID-19. Método: Revisão narrativa da literatura que selecionou artigos na base dados USA National Library of Medicine (Pubmed), no portal regional da Biblioteca Virtual em Saúde (BVS) e na biblioteca eletrônica Scientific Eletronic Library Online (Scielo) utilizando os termos de busca "Aleitamento Materno", "Coronavírus" e "Infecções por Coronavírus", e entre os termos o operador boleano AND. Na Pudmed os descritores foram "Breast Feeding", "Coronavirus" e "Coronavirus Infections", conforme o Medical Subject Headings (MeSH). Incluíramse artigos em português e publicado entre 2019-2021. Resultados: 129 artigos foram selecionados, 125 foram excluídos e três foram analisados. Conclusão: Ainda há escassez de estudos abordando a temática principalmente em português. Contudo, até o momento, as recomendações vigentes encorajam a amamentação pelas nutrizes com suspeita ou positivas para COVID-19, porém com medidas de biossegurança corretas devido aos benefícios da amamentação.

Palavras-chaves: Aleitamento materno; Coranavírus; Infecções por coronavírus

\begin{abstract}
Objective: Analyze breastfeeding during COVID-19 pandemic. Method: Narrative review of the literature that selected articles in the USA National Library of Medicine (Pubmed) database, in the regional portal of the Virtual Health Library $(\mathrm{VHL})$ and in the electronic library Scientific Electronic Library Online (Scielo) using the descriptors "Breastfeeding", "Coronavirus "and" Coronavirus Infections", and between the terms the Boolean operator AND. At Pudmed, the descriptors were "Breast Feeding", "Coronavirus" and "Coronavirus Infections", according to the Medical Subject Headings (MeSH). Articles in Portuguese and published between 2019-2021 were included. Results: 129 articles were selected, 125 were excluded and three were analyzed. Conclusion: There is still a shortage of studies addressing the theme mainly in Portuguese. However, to date, current recommendations encourage breastfeeding by breastfeeding mothers who are suspected or positive for COVID-19, but with correct biosafety measures due to the benefits of breastfeeding
\end{abstract}

Keywords: Breastfeeding; Coranavirus; Coronavirus infections.

RESUMEN | Objetivo: Analizar la lactancia materna en tiempos de la pandemia de COVID-19. Método: Revisión narrativa de la literatura que seleccionó artículos en la base de datos de la Biblioteca Nacional de Medicina de EE. UU. (Pubmed), en el portal regional de la Biblioteca Virtual en Salud (BVS) y en la biblioteca electrónica Scientific Eletronic Library Online (Scielo) utilizando los términos de búsqueda. "Lactancia materna", "Coronavirus" e "Infecciones por coronavirus", y entre los términos el operador booleano AND. En Pudmed, los descriptores fueron "Lactancia materna", "Coronavirus" e "Infecciones por coronavirus", de acuerdo con Medical Subject Headings (MeSH). Se incluyeron artículos en portugués y publicados entre 2019-2021. Resultados: se seleccionaron 129 artículos, se excluyeron 125 y se analizaron tres. Conclusión: todavía hay una escasez de estudios que aborden el tema principalmente en portugués. Sin embargo, hasta la fecha, las recomendaciones actuales fomentan la lactancia materna en madres que amamantan con sospecha o positividad de COVID-19, pero con las medidas de bioseguridad correctas debido a los beneficios de la lactancia materna.

Palabras claves: Lactancia materna; Coronavirus; Infecciones por coranavirus.

\section{Bianka Sthefany Silva}

Discente do curso de Graduação em Enfermagem da Universidade do Estado de Minas Gerais/UEMG/Unidade Passos, MG, Brasil. ORCID: 0000-0001-7981-539X

\section{Karoline Soares Chaves}

Discente do curso de Graduação em Enfermagem da Universidade do Estado de Minas Gerais/UEMG/Unidade Passos, MG, Brasil. ORCID: 0000-0003-3510-6000

\section{Gabriela da Cunha Januário}

Docente do curso de Graduação em Enfermagem da Universidade do Estado de Minas Gerais/UEMG/Unidade Passos, MG, Brasil. ORCID: 0000-0002-6425-7433

Recebido em: 09/03/2021

Aprovado em: 26/04/2021
Larissa Sales Martins Baquião

Docente do curso Técnico em Enfermagem do Instituto Federal de Educação, Ciência e Tecnologia do Sul de Minas/ IFSULDEMINAS/Campus Muzambinho, MG, Brasil.

ORCID: 0000-0002-7964-3935

\section{André Tadeu Gomes}

Discente do curso de Medicina da Faculdade Atenas. Passos, MG, Brasil.

ORCID: 0000-0002-0114-3391

\section{Glilciane Morceli}

Docente do curso de Graduação em Enfermagem da Universidade do Estado de Minas Gerais/UEMG/Unidade Passos, MG, Brasil.

ORCID: 0000-0001-8216-9931
INTRODUÇÃO

m dezembro de 2019, na cida-

- de de Wuhan na China foi identificado um novo coronavírus 2, causador da Síndrome Respiratória Aguda Grave (SARS-CoV-2) e a doença gerada pelo novo vírus foi intitulada de COVID-19. Espalhou-se rapidamente pelo território chinês ${ }^{(1,2,3)}$, e em fevereiro de 2020 foi detectado em vários países ${ }^{(1)}$, atingindo posteriormente todos os continentes $^{(3)}$ o que culminou com a declaração da Organização Mundial da Saúde (OMS) caracterizando a COVID-19 como uma pandemia, "emergência de saúde pública de importância internacional"(4) e um problema global. 
No início da pandemia a população mais acometida foi a com idade superior a $60 \operatorname{anos}^{(3)}$, porém com a rápida disseminação da COVID-19 todas as faixas etárias, condições sociais e econômicas foram atingidas, incluindo mulheres no ciclo gravídico-puerperal, contudo as repercussões tanto maternas como perinatais da COVID-19 ainda estão pouco evidenciadas na literatura em relação ao comportamento da COVID-19 nos organismos do binômio mãe/feto, assim como durante o período da amamentação(5).

O SARS-CoV-2 é transmitido diretamente através do contato próximo de pessoas, principalmente por meio de gotículas respiratórias ou indiretamente, pelo contato com superfícies ou objetos utilizados pela pessoa infectada ${ }^{(6)}$.É sabido da importância do contato mãe e filho já nos primeiros minutos após o nascimento, inclusive já com o início da amamentação, porém alguns países, em casos suspeitos ou confirmados de puérperas com COVID-19, há a separação da mãe e recém-nascido, mesmo com a recomendação da OMS da manutenção do contato e da amamentação neste período $^{(7)}$ Esta separação poderia impactar, inclusive, na redução ou ausência da oferta do leite materno.

$\mathrm{Na}$ literatura, temos informações limitadas acerca da transmissão do novo coronavírus pela amamentação e os dados estão em constante atualização, porém considera-se que a transmissão materno-infantil seja mais provável através do contato direto, pela eliminação de gotículas da mãe para o filho pelo contato próximo durante a amamentação(8), sendo necessário que as nutrizes com sintomas da COVID-19 ou com diagnóstico positivo para doença mantenham alguns cuidados essenciais durante a amamentação como a higiene assídua das mãos e uso de máscara facial ${ }^{(9)}$.

As interrupções nos serviços essenciais de saúde durante a COVID-19 afetaram gravemente a qualidade do atendimento prestado as gestantes durante o período pré-natal e isso também repercute na saúde dos filhos. Entende-se que a qualidade da assistência ao pré-natal influencia dire- tamente na saúde materno-infantil e não deve se limitar à realização de consultas e exames, pois visa uma assistência integral $^{(10)}$ As orientações, neste período, sobre os cuidados em saúde são tidas como um diferencial que contribui para uma atenção humanizada e integral ${ }^{(11)}$

Realizar ações em saúde que abordam sobre os cuidados com a gestante e recém-nascido, além de incentivar a amamentação, são essenciais, pois sabemos que muitas gestantes vivenciam este período com carência de informações, no entanto, com a recomendação de isolamento social, grupos operativos também foram suspensos, o que pode repercutir no aleitamento materno ${ }^{(12)}$.

A amamentação é considerada uma prática milenar que possui diversos benefícios imunológicos, cognitivos, socioeconômicos e nutricionais para o binômio mãe/ recém-nascido(13,14,15). Deve ser iniciada logo após o nascimento porque além da rica composição nutricional do leite materno ainda há outros componentes com propriedades imunoprotetoras que promovem e ajudam na redução de chances de infecção do neonato e também auxilia o recém-nascido contra possíveis agressões do meio ambiente ademais possui hormônios e peptídeos promotores de crescimento $^{(16,17,18)}$. Desta maneira o aleitamento materno além de fornecer ao recém-nascido todo aporte nutricional para o seu crescimento e desenvolvimento também pode ser considerado como fator protetor contra a COVID-19, devido aos seus componentes imunológicos, destacando assim a importância da amamentação durante a pandemia do novo coronavírus.

Frente ao cenário de pandemia no qual nos encontramos e devido ao incremento de novas pesquisas relacionadas à temática da amamentação por nutrizes acometidas pela COVID-19 ou com suspeita da doença, há o questionamento sobre a manutenção do aleitamento materno devido à necessidade de adoção de algumas medidas de segurança para a prevenção e controle da infecção neonatal, porém em pesquisas recentes com nutrizes ainda não foi detectado no leite materno o novo coronavírus, no entanto já foram identificados anticorpos específicos contra o mesmo nas amostras de leite de mães testadas como positivas para a doença ${ }^{(19,20)}$.

Nessa perspectiva, considerando a necessidade de acesso a conhecimentos baseados em evidências científicas que possam nortear as práticas seguras de aleitamento materno durante a pandemia da COVID-19, observa-se a relevância do presente estudo, que tem por objetivo realizar uma revisão de literatura narrativa com a finalidade de Analisar a amamentação em tempos de pandemia da COVID-19.

\section{METODOLOGIA}

Trata-se de um estudo de revisão narrativa da literatura. Este tipo de pesquisa tem como objetivo descrever e discutir o desenvolvimento de um determinado assunto sob o ponto de vista teórico.

Constituem de análise da literatura publicada nos diversos meios de divulgação cientifica, interpretação e análise crítica pessoal do autor. Este estudo tem papel fundamental no campo científico, pois permite ao leitor atualizar-se em conhecimento sobre a temática estudada ${ }^{(21)}$.

Como questão norteadora do estudo, definiu: Como está a amamentação durante a pandemia da COVID-19? Para seleção dos artigos que abordavam a temática, foram realizadas buscas sistematizadas na base de dados da USA National Library of Medicine (Pubmed), no portal regional da Biblioteca Virtual em Saúde (BVS) e na biblioteca eletrônica Scientific Eletronic Library Online (Scielo). Os termos de busca foram "Aleitamento Materno", "Coronavírus" e "Infecções por Coronavírus", todos extraídos dos Descritores em Ciências da Saúde (DECS), e entre os termos o operador boleano AND. Para as buscas na Pudmed os descritores foram "Breast Feeding", "Coronavirus" e "Coronavirus Infections", de acordo com o Medical Subject Headings (MeSH), realizadas durante o mês de março de 2021 .

Quanto aos critérios de inclusão foram selecionados artigos que abordassem 
a temática com ênfase na amamentação e a COVID-19, considerando o idioma português e trabalhos publicados nos últimos dois anos entre os anos de 2019 a 2021. Foram excluídas revisões de literatura, revisões sistemáticas, documentos/ recomendações de entidades, órgãos ou instituições e estudos que não abordassem o tema proposto.

A busca inicial na literatura resultou em uma amostra de 129 publicações. Após esta etapa, ocorreu a leitura minuciosa dos títulos e dos resumos dos estudos. Conforme os critérios de elegibilidade, três artigos foram pré-selecionados. Posteriormente, realizou-se a leitura na íntegra dos artigos, e os três foram incluídos por responderem satisfatoriamente à questão norteadora, e portanto, compuseram o conjunto final de análise desta re- visão. O fluxograma com o detalhamento das etapas de seleção das publicações está apresentado a seguir na Figura 1 .

Com relação aos aspectos éticos, por se tratar de uma revisão narrativa da literatura e pelas informações secundárias serem extraídas de artigos científicos, já publicados em bases de dados e bibliotecas virtuais, não foi necessária a autorização para utilização dos dados e nem a apreciação do Comitê de Ética em Pesquisa, conforme a Resolução Nacional 466/2012.

\section{RESULTADOS}

Foram encontrados nas bases de dados 129 estudos, sendo excluídos 126 por não obedecerem aos critérios de inclusão, resultando em três artigos selecionados para o estudo. O Quadro 1 mostra

Figura 1- Fluxograma do processo de seleção dos estudos incluidos na revisão.

\begin{tabular}{|c|c|c|}
\hline BVS & Scielo \\
\hline 37 estudos & Pestudos & Pubmed \\
\hline $\begin{array}{c}34 \text { estudos excluídos por } \\
\text { tratarem-se de documentos/ } \\
\text { recomendações. }\end{array}$ & $\begin{array}{c}\text { 2 estudos excluídos por não } \\
\text { estarem no idioma } \\
\text { português. }\end{array}$ \\
\hline 3 estudos & $\begin{array}{c}\text { Apenas um artigo se } \\
\text { enquadra nos critérios de } \\
\text { inclusão, porém o estudo já } \\
\text { foi incluído na pesquisa. }\end{array}$ \\
\hline 1 estudo & Nenhum estudo \\
\hline
\end{tabular}

Fonte: Autores

Quadro 1- Informações quanto ao título, autoria, ano de publicação e objetivo dos estudos que foram selecionados para participar da pesquisa.

\begin{tabular}{|c|c|c|c|c|c|}
\hline $\begin{array}{l}\text { Nú- } \\
\text { mero }\end{array}$ & Título & Autores & $\begin{array}{c}\text { Ano de } \\
\text { publicação }\end{array}$ & $\begin{array}{l}\text { Tipo de } \\
\text { estudo }\end{array}$ & Objetivo \\
\hline 01 & $\begin{array}{l}\text { Refletindo sobre o contexto } \\
\text { Da amamentação durante a } \\
\text { Pandemia da Covid-19. }\end{array}$ & $\begin{array}{l}\text { Dantas AC, Santos, } \\
\text { W Nascimento, AAA, } \\
\text { Oliveira LAM. }\end{array}$ & 2020 & $\begin{array}{l}\text { Estudo teórico } \\
\text { de cunho } \\
\text { reflexivo. }\end{array}$ & $\begin{array}{l}\text { Objetiva-se desenvolver uma reflexão sobre } \\
\text { as recomendações da pratica do aleita- } \\
\text { mento materno no cenário da pandemia da } \\
\text { COVID-19 com base em estudos científicos e } \\
\text { protocolos atuais publicados. }\end{array}$ \\
\hline 03 & $\begin{array}{l}\text { Ações da enfermagem nos } \\
\text { bancos de leite humano em } \\
\text { tempos de COVID-19. }\end{array}$ & $\begin{array}{l}\text { Marchiori G. RS, Alves } \\
\text { VH, Pereira AV, Vieira } \\
\text { BDG, Rodrigues D P, } \\
\text { Dulfel PA. M. et al. }\end{array}$ & 2020 & $\begin{array}{l}\text { Estudo } \\
\text { descritivo, de } \\
\text { abordagem } \\
\text { qualitativa. }\end{array}$ & $\begin{array}{c}\text { Analisar as ações das coordenações dos } \\
\text { Bancos de Leite Humano para favorecer a } \\
\text { continuidade da amamentação durante a } \\
\text { pandemia da COVID-19. }\end{array}$ \\
\hline
\end{tabular}


aleitamento e a COVID-19 e a prática da amamentação no cenário atual ${ }^{(24)}$.

Segundo orientações da OMS os benefícios da continuidade da amamentação em tempos de COVID-19 são superiores aos riscos, sendo, portanto o aleitamento materno recomendado, desde que as mulheres que desejam amamentar realizem a higiene respiratória, lavagem das mãos antes e depois de tocar no recém-nascido, e a desinfecção do ambiente sempre que houver contato ${ }^{(27)}$.

O Ministério da Saúde recomenda ainda que o uso de máscara durante a amamentação seja obrigatório e utilizado em todas as mamadas, sendo necessária sua substituição em casos de tosse ou espirro, no caso de utilização de ordenha mecânica, após o uso da máquina é necessário à higienização da mesma e após a retirada do leite, caso necessário considerar a ajuda de alguém não contaminado para ofertar o copinho ou colher do leite ordenhado ${ }^{(28)}$.

As nutrizes que não se sentirem confortáveis e não desejarem amamentar durante a infecção pela COVID-19, é recomendado que as mesma realizem a expressão do leite ou a relactação, visando a manutenção do fornecimento do leite materno depois de restabelecida da infeção pelo novo vírus ${ }^{(27)}$.

Recentemente pesquisadores analisaram amostras do leite materno, líquido amniótico, swab neonatal no nascimento e sangue do cordão umbilical, encontrando resultados negativos em todas as amostras para SARS-CoV-2, sugerindo que não ocorreu transmissão vertical da COVID-19 durante a fase tardia da gravidez ${ }^{(29)}$.

Por se tratar de um vírus recentemente descoberto, é comum o surgimento de dúvidas, medo e preocupação com relação ao aleitamento materno e os riscos para nutrizes e recém-nascidos ${ }^{(30)}$. Corroborando com esses achados, Lima et al, evidenciaram sentimentos de insegurança, medo e ansiedade vivenciados pelas lactentes quando abordadas sobre o assunto ${ }^{(25)}$.

Como intervenção os autores do estudo supracitado realizam consultorias em amamentação através das mídias sociais, já que o distanciamento é uma das medidas de precação para a pandemia. Essas lives foram importantes para a manutenção do aleitamento materno, sendo consideradas um dispositivo agregador na atenção à saúde das mulheres ${ }^{(25)}$.

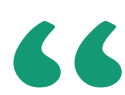

\section{Como maneira de fornecimento seguro da amamentação, a doação de leite humano também é uma alternativa importante}

Novas adaptações do banco de leite humano Como maneira de fornecimento seguro da amamentação, a doação de leite humano também é uma alternativa importante (27). Neste contexto, Marchiori et al, abordou as ações de enfermagem frente ao banco de leite humano considerando o cenário atual e como suas ações foram afetadas diretamente pela pandemia ${ }^{(26)}$.
Para evitar a disseminação do vírus e favorecer a amamentação foram necessárias adequações pela equipe de enfermagem visando minimizar o risco de contagio pela COVID-19 das nutrizes, recém-nascidos, familiares e profissionais de saúde ${ }^{(31)}$. Em relação às mudanças e adaptações que foram realizadas neste período algumas incluíram a promoção do distanciamento social, por meio da coleta domiciliar das doações de leite humano, a intensificação dos atendimentos por meio telefônico e a utilização das mídias sociais para promover o conhecimento sobre a amamentação ${ }^{(26)}$.

Como recomendação o Ministério da Saúde (MS) contraindica a doação de leite por mulheres positivas para $\mathrm{CO}$ VID-19, ou que estejam apresentando algum sintoma gripal. Apesar da recomendação do MS, ainda é fundamental que a doação seja continuada após o fim do período infeccioso, e que os profissionais que trabalham no banco de leite humano realizem as adaptações necessárias para a continuidade da assistência ${ }^{(31)}$.

\section{CONCLUSÃO}

No contexto da grave pandemia da COVID-19 que se perpetua a mais de um ano, com grande número de pessoas acometidas pelo vírus, principalmente aqui no Brasil, ainda há escassez de estudos abordando a temática principalmente em português e por isto a necessidade de continuação das investigações sobre o assunto e atualizações constantes.

Contudo, até o momento, as recomendações vigentes encorajam a amamentação pelas nutrizes com suspeita ou positivas para COVID-19 desde que sejam adotadas as medidas de biossegurança corretas, pois os benefícios da amamentação superam os possíveis riscos de contaminação do recém-nascido.

\section{Referências}

1. Zhu H, Wang L, Fang C, Peng S, Zhang L, Chang G, et al. Clinical analysis of 10 neonates born to mothers with 2019-nCoV pneumonia. Transl Pediatr
[Internet]. 2020 Feb [cited 2021 Mar 2];9(1):51-60. Available from: http:// dx.doi.org/10.21037/tp.2020.02.06 


\section{Referências}

2. Chen N, Zhou M, Dong X, Qu J, Gong F, Han Y, et al. Epidemiological and clinical characteristics of 99 cases of 2019 novel coronavirus pneumonia in Wuhan, China: a descriptive study. The Lancet [Internet]. 2020 Feb [cited 2021 Mar 2];395(10223):507-13. Available from: http://dx.doi.org/10.1016/ s0140-6736(20)30211-7

3. Li Q, Guan X, Wu P, Wang X, Zhou L, Tong Y, et al. Early transmission dynamics in Wuhan, China, of novel coronavirus-infected pneumonia. $N$ Engl J Med. 2020;382:1199-207. Available from: http://dx.doi.org/ 10.1056/NEJMoa2001316

4. WHO. Coronavirus disease 2019 (COVID-19) Situation Report-57.2020. https://www.who.int/docs/default-source/coronaviruse/situation-reports/ 20200428-sitrep-99-covid-19.pdf?sfvrsn=119fc381_2 (acessado em 02 de marçol de 2021).

5. Stanojevi囚 M. Are Covid-19-positive mothers dangerous for their term and well newborn babies? Is there an answer? Journal of Perinatal Medicine [Internet]. 2020 Jun 25 [cited $2021 \mathrm{Mar} 2$ ]; 48(5):441-5. Available from: http:// dx.doi.org/10.1515/jpm-2020-0186

6. Brasil. Ministério da Saúde. Secretaria de Vigilância em Saúde. Departamento de Análise em Saúde e Doenças não Transmissíveis. Guia de vigilância epidemiológica Emergência de saúde pública de Importância nacional pela Doença pelo coronavírus 2019 - covid-19 [recurso eletrônico] / Ministério da Saúde, Secretaria de Vigilância em Saúde. - Brasília : Ministério da Saúde, 2021.

7. Nova pesquisa destaca riscos de separar recém-nascidos de suas mães durante pandemia de COVID-19 - OPAS/OMS | Organização Pan-Americana da Saúde [Internet]. [cited 2021 Mar 22]. Available from: https://www.paho.org/ pt/noticias/16-3-2021-nova-pesquisa-destaca-riscos-separar-recem-nascidos-suas-maes-durante-pandemia

8. Center for Disease Control and Prevention (CDC). Coronavirus 2019 Pregnancy \& Breastfeeding. Protect yourself and your family from COVID-19 [Internet]. Atlanta: CDC; 2020; [acesso em 2021 Mar 02]. Disponível em: https://www.cdc.gov/coronavirus/2019-ncov/need-extra-precautions/ pregnancy-breastfeeding.html?CDC_AA_refVal=https $\% 3 \mathrm{~A} \% 2 \mathrm{~F} \% 2 \mathrm{Fwww} . c \mathrm{dc}$. gov\%2Fcoronavirus $\% 2$ F2019-ncov\%2Fprepare\%2Fpregnancy-breastfeeding. Html

9. Chaves R, Lamounier J, Santiago L. Aleitamento materno e terapêutica para a doença coronavírus 2019 (COVID-19). Resid Pediatr [Internet]. 2020 [cited 2021 Mar 2];10(2). Available from: http://dx.doi.org/10.25060/residpediatr-2020.v10n2-323

10. de Souza Sotto Mayor M, S. C. Herrera SD, Queren Araujo M, Martins dos Santos F, Vilela Arantes R, Araújo de Oliveira N. Avaliação dos indicadores da assistência pré-natal em unidade de saúde da família, em um município da Amazônia Legal. Revista Cereus. 2018 May 14;10(1):91-100.

11. Gaíva MAM, Palmeira EWM, Mufato LF. Women's perception of prenatal and delivery care in cases of neonatal death. Escola Anna Nery. 2017 Aug 17;21(4).

12. Baquião LSM, Ângelo ST, Costa AMB, Dias ALG, Constâncio JVB. Práticas educativas a gestantes em situação de vulnerabilidade social. Brazilian Journal of Development. 2020;6(4):16903-7.

13. Chaves RG, Lamounier JA, César CC. Factors associated with duration of breastfeeding. J Pediatr $2007 ; 83(3): 241-246$

14. Moraes LL, Campos LA, Ferrari DVJ, Saeki EK, et al. Impacto da idade materna na acidez do colostro de nutrizes em maternidade do interior do Estado de São Paulo. 2019; 29(2):153-160. DOI http://doi.org/10.7322/jhgd. v29.9414

15. Pereira Depolito SC, Lima de Moraes L, Garcia Siqueira W, Sales Martins Baquião L, Da Cunha Januário G, Morceli G. Atuação da equipe de enfermagem frente ao desmame precoce: uma revisão narrativa. SaudColetiv (Barueri) [Internet]. 2020 Sep 3 [cited $2021 \mathrm{Mar} 2$ ];(55):2915-24. Available from: http://dx.doi.org/10.36489/saudecoletiva.2020v10i55p2915-2924

16. Ferrari DV de J, Polettini J, de Moraes LL, de Campos LA, da Silva MG, Saeki EK, et al. Profile of pro-inflammatory cytokines in colostrum of nursing mothers at the extremes of reproductive age. PLoS ONE [Internet]. 2020 Jun 16 [cited 2021 Mar 2];15(6):e0231882. Available from: http://dx.doi. org/10.1371/journal.pone.0231882

17. Rajewska A, Mikołajek-Bedner W, Lebdowicz-Knul J, Sokołowska M, Kwiatkowski S, Torbé A. COVID-19 and pregnancy - where are we now? A review. Journal of Perinatal Medicine [Internet]. 2020 Jun 25 [cited $2021 \mathrm{Mar}$ 2];48(5):428-34. Available from: http://dx.doi.org/10.1515/jpm-2020-0132 18. Moraes Martins AB, Vieira IC, Polettini J, Lima de Moraes L, Garcia Siqueira W, Morceli G. Aleitamento materno e seu conhecimento por alunos de enfermagem. Nursing (São Paulo). 2020 Aug 25;23(267):4430-41.

19. World Health Organization (WHO). New FAQs address healthcare workers questions on breastfeeding and COVID-19 [Internet]. Geneva:WHO; 2020; [acesso em $2021 \mathrm{Mar}$ 02]. Disponivel em: https://www.who.int/news-room/ detail/28-04-2020-new-faqs-address-healthcare-workers-questions-on-breastfeeding-and-covid-193.

20. Center for Disease Control and Prevention (CDC). Coronavirus 2019 Pregnancy \& Breastfeeding. Protect yourself and your family from COVID-19 [Internet]. Atlanta: CDC; 2020; [acesso em 2021 Mar 02]. Disponível em: https://www.cdc.gov/coronavirus/2019-ncov/need-extra-precautions/ pregnancy-breastfeeding.html?CDC_AA_refVal=https $\% 3$ A $\% 2 F \% 2 F w w w . c d c$. 21. Rother ET. Revisão sistemática $X$ revisão narrativa. Acta Paul. Enferm [Internet]. 2007 [cited $2021 \mathrm{Mar} 1$ ]; 20(2):5-6. Available from: https://www. scielo.br/scielo.php?script=sci_arttext\&pid=S010321002007000200001 22. World Health Organization (WHO). Infection prevention and control during health care when COVID-19 is suspected [Internet]. 2020 mar [cited 2020 abr 23]. Available from: https://www.who.int/publications-detail/infection-prevention-and-control-during-health-care-when-novel-coronavirus-(ncov)-infection-issuspected-20200125.

23. Centers for Disease Control and Prevention (CDC). Coronavirus Disease 2019 [Internet]. 2020 [cited $2020 \mathrm{abr}$ 24]. Available from: https://www.cdc. gov/coronavirus/2019-ncov/daily-life-coping/managing-stress-anxiety.html. 24. Dantas AC.; Santos, W Nascimento, AAA, Oliveira LAM. Refletindo sobre o contexto da amamentação durante a Pandemia da Covid-19. Enferm. Foco [Internet]. 2020 [cited 2021 Mar 3]; 11 (Esp. 2): 236-239. Available from: http://biblioteca.cofen.gov.br/refletindo-sobre-contexto-amamentacao-durante-pandemia-covid-19/

25. Lima ACMCC, Chaves MAF L, Oliveira M G, Lima SAFCC, Machado M. MT; Oriá MOB. Consultoria em amamentação durante a pandemia COVID-19: relato de experiência. Esc Anna Nery [Internet]. 2020 [cited 2021 Mar 2];24(spe):e20200350. Available from: https://www.scielo.br/scielo.php?pi$\mathrm{d}=\mathrm{S} 141481452020000500602 \&$ script $=$ sci_arttext

26. Marchiori G. RS, Alves VH, Pereira AV, Vieira B. DG, Rodrigues D P, Dulfel PA. M. et al. Ações da enfermagem nos bancos de leite humano em tempos de COVID-19. Rev Bras Enferm [Internet]. 2020 [cited 2021 Mar 2];73(Suppl 2): e20200381. Available from: http://dx.doi.org/10.1590/0034-7167-20200381 e20200381

27. World Health Organization (WHO). Q\&A: Pregnancy, childbirth and COVID-19 [Internet]. 2020 [cited 2020 abr 24]. Available from: https://www. who.int/news-room/q-a-detail/q-a-on-covid-19-pregnancy-childbirth-and-breastfeeding.

28. Ministério da Saúde (BR). Nota técnica n7/2020-DAPES/SAPS/MS: Trata-se de avaliação de medida para o enfrentamento da situação de emergência em saúde publica decorrente do Coronavírus (Covid-19) [Internet]. Brasília: Ministério da Saúde; 2020 [cited 2020 abr 25]. Available from: https://portaldeboaspraticas.iff.fiocruz.br/biblioteca/covid-19-e-amamentacao-nota-tecnica-no-7-2020-dapes-saps-ms/.

29. Belasco AGS, Fonseca CD. Coronavírus 2020. Rev Bras Enferm [Internet]. 2020 [cited 2021 Mar 2];73(2):e2020n2. Available from: doi: 10.1590/00347167-2020730201.

30. Centers for Disease Control and Prevention (CDC). Coronavirus Disease 2019 [Internet]. 2020 [cited 2021 Mar 23]. Available from: https://www.cdc. gov/ coronavirus/2019-ncov/daily-life-coping/managing-stress-anxiety.html. 31. Belasco AGS, Fonseca CD. Coronavírus 2020. Rev Bras Enferm [Internet]. 2020 [cited 2021 Mar 2];73(2):e2020n2. Available from: doi: 10.1590/00347167-2020730201. 